\title{
Hypomagnesemia signals nonrecovery of renal function in AKI
}

Published online: 17 July 2012

(C) Springer Healthcare 2012

MedWire News: Researchers have identified hypomagnesemia as a risk factor for nonrecovery of renal function among critically ill patients with acute kidney injury (AKI).

Hypomagnesemia, defined as a total serum magnesium concentration below $0.70 \mathrm{mmol} / \mathrm{L}$, is a frequent electrolyte abnormality that occurs in over $12 \%$ of all hospitalized patients and $60-65 \%$ of those in intensive care.

Cassiana Mazon Fraga (Universidade do Extremo Sul Catarinense, Santa Catarina, Brazil) and colleagues investigated whether measuring daily magnesium serum levels among 232 patients admitted to an intensive care unit (ICU) could help identify those who fail to recover renal function.

In all, 118 patients developed AKI according to the Risk, Injury, Failure, Loss and End-stage kidney disease (RIFLE) criteria, 71 recovered from AKI, and 88 patients died.

The overall prevalence of hypomagnesemia among patients was $63 \%$, with 97 of those admitted to the ICU already presenting with the condition. The average duration of hypomagnesemia during ICU stay was 3.1 days.

The prevalence of hypomagnesemia was similar between patients with and without AKI, at $47 \%$ and $62 \%$, respectively, and the presence or absence of hypomagnesemia was not associated with AKI.

However, it was associated with the recovery of renal function, occurring in just 30\% of patients who recovered renal function, defined as an absence of AKI by RIFLE criteria over a 48-hour period or at ICU discharge, compared with $70 \%$ of those who did not.

Multivariate analysis that took into account sepsis and RIFLE classification showed that hypomagnesemia was a significant risk factor for nonrecovery of renal function, with ICU mortality similar in patients with and without hypomagnesemia.

"Hypomagnesemia is an independent risk factor for non-recovery of renal function among AKI patients in the ICU," conclude the authors in Nephrology Dialysis Transplantation.

By Ingrid Grasmo

\section{Reference}

Nephrol Dial Transplant 2012; Advance online publication 